\title{
Wood physical properties, color, decay resistance and stiffness in Tectona grandis clones with evidence of genetic control
}

\author{
By R. Moya*), J. D. MaríN ${ }^{1)}$, O. Murillo ${ }^{2)}$ and L. LeANdro ${ }^{3)}$
}

(Received $5^{\text {th }}$ December 2012)

\begin{abstract}
Tectona grandis (teak) plantations are being produced with trees from genetic improvement programs, including clonal selection. However, limited information about inheritance of wood properties is available. For studying genetic parameters of $T$. grandis wood properties and stem diameter two 10-yr-old trials were studied involving nominally 2 sites $\mathrm{x} 20$ clones $\mathrm{x} 3$ replicates $\mathrm{x} 1$ ramet. Clonal variation was observed in: heartwood $(H W P)$, pith $(P P)$ and bark $(B P)$ percentages; green moisture content (IMC) and density $(G D)$; tangential $(T S)$, radial $(R S)$ and volumetric $(V S)$ shrinkage and ratio tangential/radial: shrinkage (ratio $T / R$ ); $\mathrm{L}^{*} \mathrm{a}^{*} \mathrm{~b}^{*}$ color parameters; and stiffness and specify gravity $(S G)$. Some wood properties evidenced strong across-sites genotypic control. The higher broad-sense heritability estimates (over 0.36) were for PP, IMC, SG, TS, VS and $L^{*}, a^{*}$, and $b^{*}$ color coordinates and stiffness. Low and non-significant values were estimated for $H W P, B P, G D$, $R S$, ratio $T / R$ and decay resistance of sapwood and heartwood to Trametes versicolor and Pycnoporus sanguineus. However, HWP showed marked clone $\mathrm{x}$ site interaction. Phenotypic and tentatively estimated genotypic correlations indicate that selecting faster-growing clones will improve some important wood properties, such as increasing $H W P$, and decreasing $P P$ and $B P$, without affecting other wood properties. Selecting clones for denser wood, as expected, improved stiffness, should decrease IMC and affect negatively $b^{*}$ (yellow/blue) color. Clone selection with lower $L^{*}$ values could increase redness $\left(a^{*}\right)$ of wood.
\end{abstract}

Key words: teak, genetic parameters, broad-sense heritability, genotypic correlations, wood properties, heartwood color, wood decay.

\section{Introduction}

Among tropical forest species, teak (Tectona grandis L.f.) has become one of the most important in the market due to its physical, mechanical and aesthetic timber properties. Its excellent workability properties, strong decay resistance, excellent dimensional stability, and

\footnotetext{
1) Universidad del Tolima, Faculta de Ingeniería Forestal, B Santa Helena A.A. 546 - Ibagué, Colombia. E-Mail: jdmm086@hotmail.com.

2) Instituto Tecnológico de Costa Rica, Escuela de Ingeniería Forestal, Apartado 159-7050, Cartago, Costa Rica. E-Mail: omurillo@itcr.ac.cr.

3) Universidad de Costa Rica, Facultad de Ingeniería, Costa Rica E-Mail: lalezu76@gmail.com.

*) Corresponding author: RóGer MoyA. Instituto Tecnológico de Costa Rica, Escuela de Ingeniería Forestal, Apartado 159-7050, Cartago, Costa Rica. E-Mail: rmoya@itcr.ac.cr.
}

highly desired wood color, have allowed this tree species to become one of the most planted and marketable ( $\mathrm{GOH}$ and Monteuuis, 2005). Today teak is being planted in Costa Rica as a solid-wood crop, with sitting, site preparation, breeding programs and management all rapidly improved in the last few years (MURILLO, 2005). One of the most practical and effective strategies in improving productivity has been developing new plantations through clonal forestry (XAVIER and OTONI, 2009). Clonal deployment from properly developed breeding programs allows more uniform plantations, higher yield and shorter rotations, among other benefits (MonTEUUIS and GoH, 1999; MonteuUis and MaItRe, 2007). More advanced breeding generations may provide better clone-to-site matching and disease resistance (XAVIER and OTONI, 2009).

Most teak breeding efforts have only addressed volume growth, stem quality, branch exertion angle, and buttress reduction, among other external traits $(\mathrm{GOH}$ and Monteuuis, 2005; CAllister and Collins, 2008). Therefore, there is a major need to incorporate wood traits into breeding and plantation management procedures, in order to improve lumber prices in international markets. Some reports include heritability estimates for traits like wood quality, heartwood diameter, bark thickness, wood calcium and silica contents, and wood density and durability (KJÆR et al., 1999 and 1996; VARGHESE et al., 2000). However, none of all these investigations has involved clonal material, but instead regular provenance and progeny tests.

Today, wood color is being considered as another important attribute in teak lumber coming from plantations, especially at the market (THULASIDAS et al., 2006). Even though there are no reports in literature about teak on genetic control for wood color, other investigations report mixed findings for other tree species (RINK, 1987; Sotelo et al., 2008; HANNRUP et al., 2004; Mosedale et al., 1996). Other wood properties, like shrinkage, density, durability and mechanical properties, have received great attention in breeding and clonal forestry (ZoBEL and JETT, 1995), but again, very little in the case of teak. Recent investigations on breeding for stiffness have shown that, using ultrasound techniques, it is possible to get the proper information for selecting trees with sufficient effective heritabilities (SoLORZANO et al., 2012b).

This research was aimed at generating new information about teak wood properties and their genetic control, especially in the following traits: specific gravity, green density, some tree traits (heartwood percentage 
and bark thickness), wood shrinkage (tangential, radial and volume) color parameters (through CIE L* $\mathrm{a}^{*} \mathrm{~b}^{*}$ system), fungal decay, and stiffness.

\section{Material and Methods}

Study site description: The study involved in two teak clonal trials located in two different sites in northern Costa Rica. Site 1 (Garza) has an average rainfall of $1594 \mathrm{~mm}$, a mean annual temperature of $26^{\circ} \mathrm{C}$, and a 4month dry season without rain. The soil is a loam, is moderately acid to neutral ( $\mathrm{pH} \mathrm{6-7)}$ and moderately fertile but with low organic matter content, and the slope is less than $3 \%$. Site 2 (Peñas Blancas) has an average rainfall of $1745 \mathrm{~mm}$, a mean temperature of $27^{\circ} \mathrm{C}$, and a 5 -month dry season without rain. The soil has a loam to

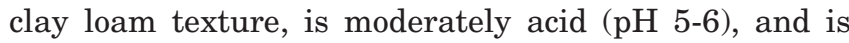
moderately fertile soil with moderate organic matter content, while the slope is less than $3 \%$.

Tree selection and stand conditions: Plus trees were selected as part of a breeding program at Precious Woods company throughout more than 2000 planted ha in northeastern Costa Rica, as well as from provenance trials (mostly asean origin) established in several sites. Therefore, selections comprehend quite a large genetic variation. Selection criteria were based mostly on growth rate and stem quality. Selected trees were cloned directly by taking stem sprouts and, in some cases, collecting stump sprouts after felling them down. Propagules (cuttings) were brought to greenhouse facilities and rooted (Víquez and PÉREZ, 2005). Once all plus trees were vegetatively propagated, small clonal gardens were established within a greenhouse and utilized in multiplication of all materials for establishing genetic tests. Clonal trial age was 10 years at the time of this investigation, which corresponds to the second commercial thinning. Clonal trial cites were used by cattle in the past. Originally, the experiment included 40 clones planted in five blocks, with two single ramets randomly distributed within each block (Figure 1a). This resulted in a total of 80 trees per block at $3 \times 3 \mathrm{~m}$ spacing with uniform spacing between road and columns. The trials had a 50\% thinning at age 5, but including mortality, only 20 from the original 40 clones remained with most of their trees alive in good conditions at age 10. Therefore, the clones that were included in this investigation were the ones with the largest number of standing trees at all three blocks within each of the two sites, aiming to ensure some statistical balance in data. Thus, clones with the best possible representativeness in both trials was their main selection criteria for this investigation. Some more detailed information about the sampled trees was included in Table 1.

Sampling procedures: One tree from each clone in the first three blocks at each site was selected, that is, 1 tree per clone $\mathrm{x} 2$ sites $\mathrm{x} 3$ replicates (a total of 6 trees per clone). Trees felled had straight trunks, low branching thickness and near 90o insertion angle, and non visible disease or pest symptoms. The north-facing side of each tree was marked before being felling down. Then a $100 \mathrm{~cm}$-long log sample was taken from $0.3 \mathrm{~m}$ to $1.3 \mathrm{~m}$ (Figure 1b). Additionally, two $3 \mathrm{~cm}$-thick discs were obtained at DBH. A full-length $3 \mathrm{~cm}$-thick diametric section was cut from the log from north to south (Figure 1b). These samples were conditioned at $22^{\circ} \mathrm{C}$ and at a relative humidity of $66 \%$, in order to obtain a $12 \%$ equilibrium moisture content. Several wood samples exhibiting knots, evidence of rot, or any other defects, had to be discarded since they could interfere with ultrasonic waves used within the probe.

Heartwood, pith and bark percentage determination: These traits were determined on each stem cross-section. Heartwood and pith diameter were obtained from the average of two complete cross-sectional measurements (direction north-south and east-west). The total mean diameter (with and without bark) was calculated following the same procedure. Bark thickness was defined as half the difference between inside and outside-bark diameters. The sectional area of each component was determined as an assumed circle and the
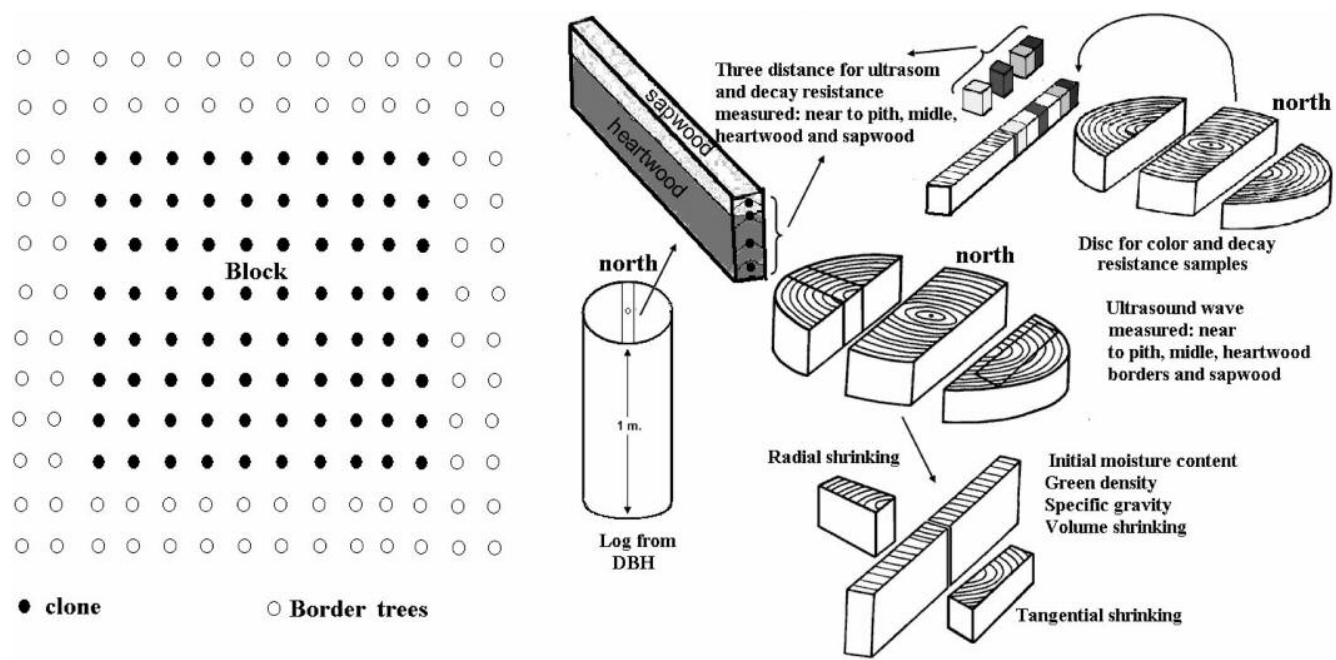

Figure 1. - Wood probes sampling procedure for testing wood properties in teak clonal investi gation. 
Table 1. - Mean diameter at breast height $(1.3 \mathrm{~m})$ and total heights for the 20 teak clones at the two sites.

\begin{tabular}{cccccc}
\hline & Gar/a & \multicolumn{3}{c}{ Pefias Blancas } \\
\hline Clone & $\begin{array}{cccc}\text { Dbh } \\
(\mathrm{cm})\end{array}$ & $\begin{array}{c}\text { Total height } \\
(\mathrm{m})\end{array}$ & $\begin{array}{c}\text { dbh } \\
(\mathrm{cm})\end{array}$ & $\begin{array}{c}\text { lotal } \\
\text { hcight } \\
(\mathrm{m})\end{array}$ & $\begin{array}{c}\text { Crown } \\
\text { height } \\
(\mathrm{m})\end{array}$ \\
\hline 1 & 15.3 & 17.7 & 23.1 & 17.2 & 6.6 \\
2 & 15.7 & 16.3 & 19.0 & 16.0 & 6.8 \\
3 & 14.9 & 13.9 & 19.8 & 18.9 & 7.3 \\
4 & 21.1 & 18.9 & 21.1 & 19.5 & 7.8 \\
5 & 20.6 & 17.1 & 22.6 & 19.6 & 7.17 \\
6 & 18.1 & 19.1 & 21.8 & 17.6 & 5.9 \\
7 & 20.1 & 19.3 & 23.0 & 19.3 & 7.4 \\
8 & 18.1 & 19.8 & 23.6 & 18.5 & 5.5 \\
9 & 20.0 & 15.8 & 27.3 & 14.8 & 10.7 \\
10 & 20.1 & 19.7 & 22.5 & 22.0 & 9.7 \\
11 & 21.8 & 16.6 & 22.2 & 18.9 & 7.2 \\
12 & 19.5 & 18.7 & 20.6 & 19.3 & 5.2 \\
13 & 18.2 & 17.1 & 22.7 & 19.0 & 5.7 \\
14 & 20.1 & 16.9 & 24.2 & 17.9 & 7.7 \\
15 & 22.6 & 18.1 & 29.9 & 19.7 & 6.7 \\
16 & 22.5 & 16.7 & 22.1 & 14.4 & 11.3 \\
17 & 18.4 & 18.0 & 22.4 & 17.9 & 7.0 \\
18 & 18.3 & 18.5 & 21.7 & 17.2 & 6.0 \\
19 & 22.2 & 18.6 & 24.4 & 19.0 & 6.8 \\
20 & 19.4 & 16.2 & 22.3 & 17.6 & 5.6 \\
\hline Average & 19.4 & 17.7 & 22.8 & 18.2 & 7.2 \\
\hline
\end{tabular}

corresponding percentages calculated. Heartwood percentage $(H W P)$ was obtained by the average of two heartwood diameters related to total inside bark-area. Pith percentage $(P P)$ and bark percentage $(B P)$ were then obtained through its proportion with respect to total inside-bark disc area.

Determining ultrasound velocity and dynamic stiffness: Longitudinal ultrasound velocity measurements were taken on the diametric boards cut from the logs along a radial transect at four distances from the pith (near to pith, midway between pith and sapwood/heartwood boundary, sapwood/heartwood boundary, and halfway into sapwood thickness). The time required by the wave to travel from one end of the board to the other was measured twice at each distance. Tests were conducted using SYLVATESTDUO ultrasound equipment with two $22 \mathrm{kHz}$ transductors. This device was set to four readings per measurement. Ultrasound velocity was calculated (Equation 1) and dynamic stiffness (Ed) by Equation 2. In order to calculate density, a $2 \times 2 \times 2 \mathrm{~cm}$ sample was extracted from where ultrasound velocity was measured. This sample was used to calculate weight and volume using the water displacement method as specified by D2395 (ASTM, 2003).

$$
\begin{aligned}
& V=\frac{L}{T} \\
& E d=V^{2} \times d \times 10^{-6}
\end{aligned}
$$

Where: $\mathrm{V}=$ ultrasound velocity in $\mathrm{m} \mathrm{s}^{-1}, \mathrm{~L}=$ sample length in meters, $\mathrm{T}=$ time required by ultrasound wave to travel from one end of board to the other in $\mu \mathrm{s}$, $E d=$ dynamic stiffness in GPa and d = wood density in $\mathrm{kg} \mathrm{m} \mathrm{m}^{-3}$.

Wood color determination: In order to determine wood color, a cross-section was taken at DBH and a $3 \mathrm{~cm}$-wide diametric section was cut (Figure $1 b$ ). Then a $2 \times 2 \times 2 \mathrm{~cm}$ sample was produced at the same four distances (near to pith, midway between pith and sapwood/heartwood boundary, sapwood/heartwood boundary, and halfway into sapwood thickness) from the pith as for ultrasound velocity determination. A nominal total of 960 specimens (8 per cross-section x 3 replicates x 20 clones x 2 sites) were obtained from the sampled clones. Wood color was determined on the block's tangential face in accordance with ASTM D-2244 (ASTM, 2005). A HunterLab MiniScan ${ }^{\circledR}$ XE Plus spectrophotometer was used. Measurements were taken at room temperature and color characteristics were determined using CIELab system (parameters $L^{*}, a^{*}$, and $b^{*}$ ). Measurement range was from 400 to $700 \mathrm{~nm}$, with a $13 \mathrm{~mm}$ aperture at the measurement point. Specular component (SCI mode) was included to observe reflection at a $10^{\circ}$ angle, which is normal for this specimen surface (D65/10), as well as a $2^{\circ}$ field of vision (standard observer, CIE 1931), and standard D65 illumination (corresponds to daylight at $6500 \mathrm{~K}$ ). According to HUNTERLAB (1995), CIELab color system estimates wood color in three coordinates: $L^{*}$ for lightness represents the position on the black-white axis ( $L=0$ for black, $L=100$ for white), $a^{*}$ for chroma value and defines the position on the red-green axis (+100 values for red shades, -100 values for green shades), and, 
$b^{*}$ for chroma value and defines the position of yellow-blue axis (+100 values for yellow shades, -100 values for blue shades).

Decay resistance: Wood specimens measuring $2.5 \mathrm{x}$ $2.5 \times 2.5 \mathrm{~cm}$ were obtained from each wood probe. White-rot fungi Trametes versicolor L. Fr. and Pycnoporus sanguineus (L.) Merrill were utilized in testing natural decay resistance following ASTM Standard D2017-81 (ASTM, 2003). The relative resistance of each test block to decay was measured as a percentage loss in oven-dry weight during 16 -week exposure to fungi. Therefore, four wood characteristics were determined: heartwood loss weight under T. versicolor ( $T$ V-HRW) and under P. sanguineus (PS-HRW); and sapwood loss weight under $T$. versicolor and $P$. sanguineus, coded as $T V$-SAP and $P S-S A P$ respectively.

Wood physical properties determination: Another group of wood physical properties were determined for each clone, based on the second cross-section at $D B H$. These properties were: radial shrinkage $(R S)$, tangential shrinkage (TS) and total volume shrinkage (VS), ratio of tangential shrinkage/radial shrinkage (Ratio $T / R$ ), wood density at green condition $(G D)$, initial or green moisture content $(I M C)$ and specific gravity $(S G)$. The rest of wood samples from each clone were obtained as shown in Figure 1b. Green weight and volume from each subsample were determined according to norm D-2395-02
(ASTM, 2003). Wood samples were oven-dried at 1050 for 24 hours, and then reweighed. This final oven-dried weight was utilized in order to determine $G D, V S, I M C$, and $S G$. Both, $T S$ and $R S$ were determined according to ASTM D-2395-02.

Statistical analyses: $H W P, P P$ and $B P, R S, T S$ and $V S$ were assessed based on single-tree values. All other wood properties were estimated based on a mean of two $(I M C, G D, S G$ ) or four samples per single tree (color parameters, stiffness and weight loss due to fungi). The individual-tree coefficient of variation $(C V)$ was estimated for each wood property and on each site. It was estimated as the ratio of the standard deviation to the mean. Before analysis of variance was performed, data were checked for normality of distribution and homogeneity of variances of data. $P P, R S, G D, S G, E D, T S$, $V S$, ratio $T / R, T V-S A P$ and $P S-S A P$ presented problem with normality and homogeneity of variance. Then logarithmic transformations were applied in $P P, R S, T S$ and $V S$, ratio $T / R, T V$-SAP and color coordinate $a^{*}$. GD was transformed to $\mathrm{X}^{2} ; S G$ was transformed to its inverse $(1 / \mathrm{X})$; stiffness $(E d)$ was transformed to its square root, and $P S-S A P$ to $\mathrm{X}^{1.5}$.

Joint analysis on both sites was performed using SELEGEN REML/BLUP software (RESENDE, 2002). The statistical model is presented in Equation 3, where, "Y" is the data vector, " $\mathrm{r}$ " is a repetition effects vector

Table 2. - Phenotypic variation in replicated 10-year-old teak clonal test at the two sites $(N=110)$.

\begin{tabular}{|c|c|c|c|c|c|}
\hline \multirow{2}{*}{$\begin{array}{c}\text { Wood } \\
\text { properties }\end{array}$} & \multirow{2}{*}{$\begin{array}{c}\text { Both site } \\
\text { Mcan (CV) }\end{array}$} & \multicolumn{2}{|c|}{ Garza } & \multicolumn{2}{|c|}{ Peñas Blancas } \\
\hline & & Mcan $(\mathrm{CV})$ & $\begin{array}{c}\text { Lowest and } \\
\text { highest } \\
\text { Value }\end{array}$ & Mcan (CV) & $\begin{array}{l}\text { Lowest and } \\
\text { highest } \\
\text { value }\end{array}$ \\
\hline$D B H$ & $19.8(15.2)$ & $19.4(15.7)$ & $12.9-25.9$ & $20.3(14.6)$ & $14.0-28.7$ \\
\hline$H W W^{\prime}$ & $38.3(19.5)$ & $37.2(21.2)$ & $16.5-55.3$ & $39.5(17.4)$ & $22.9-54.1$ \\
\hline$B P^{\prime}$ & $19.2(25.0)$ & $16.5(29.5)$ & $9.3-28.3$ & $22.0(12.1)$ & $15.9-27.3$ \\
\hline$P P$ & $0.162(31.6)$ & $0.17(55.7)$ & $0.02-0.48$ & $0.15(70.7)$ & $0.03-0.51$ \\
\hline$M C(\%)$ & $104.3(23.3)$ & $87.9(23.4)$ & $45.2-128.7$ & $121.4(11.6)$ & $89.8-158.0$ \\
\hline$G D\left(\mathrm{~g} / \mathrm{cm}^{3}\right)$ & $1.04(10.4)$ & $0.9](10.7)$ & $0.60-1.19$ & $1.18(4.2)$ & $0.90-1.21$ \\
\hline$S O$ & $0.48(8.0)$ & $0.50(7.2)$ & $0.43-0.59$ & $0.49(8.7)$ & $0.41-0.62$ \\
\hline$V S(\%)$ & $7.2(18.9)$ & $6.9(15.1)$ & $4.7-9.8$ & $7.5(21.0)$ & $4.2-12.2$ \\
\hline$T S(\%)$ & $4.2(19.3)$ & $3.6(18.6)$ & $2.5-5.8$ & $4.7(10.8)$ & $3.4-6.0$ \\
\hline$R S(\%)$ & $2.7(29.8)$ & $2.1(17.1)$ & $1.3-3.1$ & $3.4(18.2)$ & $2.1-4.6$ \\
\hline Ratio $T / R$ & $1.5(19.0)$ & $1.7(14.9)$ & $1.0-2.1$ & $1.4(19.9)$ & $1.0-2.2$ \\
\hline$L^{*}$ & $62.7(5.7)$ & $63.3(6.1)$ & $51.6-72.4$ & $62.0(5.1)$ & $54.8-70.0$ \\
\hline$a^{*}$ & $9.5(10.6)$ & $9.0(9.2)$ & $7.1-10.5$ & $9.9(9.8)$ & $7.7-11.7$ \\
\hline$b^{*}$ & $28.6(6.5)$ & $27.8(5.9)$ & $23.0-31.1$ & $29.5(5.6)$ & $25.2-33.2$ \\
\hline$E d(\mathrm{GPa})$ & $12.8(13.7)$ & $13.2(14.8)$ & $9.3-16.5$ & $12.4(11.5)$ & $9.2-15.1$ \\
\hline$T V-H R W(\%)$ & $16.9(73.4)$ & $27.6(26.8)$ & $44.5-52.5$ & $5.9(72.5)$ & $1.3-18.6$ \\
\hline$P S-H R W(\%)$ & $7.3(40.7)$ & 5. $(54.9)$ & $1.2-11.8$ & $9.1(64.0)$ & $1.4-26.8$ \\
\hline$T V-S A P(\%)$ & $30.5(41.1)$ & $39.3(15.6)$ & $23.3-52.3$ & $21.5(51.3)$ & $3.5-46.4$ \\
\hline$P S-S A P(\%)$ & $15.1(51.3)$ & $10.3(51.2)$ & $1.2-23.8$ & $20.0(33.6)$ & $2.8-43.8$ \\
\hline
\end{tabular}

$C V=$ coefficient of variation, $D B H=$ diameter at breast height, $H W P=$ heartwood percentage, $B P=$ bark percentage, $P P=$ pith percentage, $I M C=$ initial moisture content, $G D=$ green density, $S G=$ specific gravity, $V S=$ Volume shrinkage, $T S=$ Tangential shrinkage, $R S=$ Radial shrinkage, Ratio $T / R=$ relation tangential and radial shrinkage, " $L^{*}$ ", " $a$ " " and " $b$ " are color coordinates, $E d=$ stiffness, $T V-H R W$ = weight loss with Trametes versicolor fungi in heartwood, $P S-H R W=$ weight loss with Pycnoporus sanguineus fungi in heartwood, TV-SAP = weight loss with Trametes versicolor fungi in sapwood, PS-SAP = weight loss with Pycnoporus sanguineus fungi in sapwood. 
(assumed as random) and added to general mean value, "a" is an individual additive genetic effects vector (assumed as random), "p" is a plot effects vector (assumed as random), " $i$ " is the genotype $\mathrm{x}$ environment interaction effects vector (random), and "e" is the residual vector (random). Capital letters denote incidence matrices for mentioned effects. Vector " $r$ " includes all repetitions in both locations (adjusting repetition $\mathrm{x}$ location combinations). In this case, site effects and repetitions within locations are considered.

$$
\mathrm{Y}=\mathrm{Xr}+\mathrm{Za}+\mathrm{Wp}+\mathrm{Ti}+\mathrm{e},
$$

Variance component estimates were obtained using model 23 of SELEGEN software (Randomized Block Design with three repetitions and established at two locations, RESENDE, 2002). Clonal heritability was estimated for all individual wood traits based on equation 4:

$$
\hat{H}^{2}=\frac{\hat{\sigma}_{c}^{2}}{\hat{\sigma}_{c}^{2}+\hat{\sigma}_{c^{*} s}^{2}+\hat{\sigma}_{e}^{2}}
$$

where $\hat{\sigma}_{c}^{2}, \hat{\sigma}_{c^{*} s}^{2}$ and $\hat{\sigma}_{e}^{2}$ are respectively variance components for clonal, GxE interaction (clone by site), and error or residual effects. The Pearson phenotypic (clonal-means) correlation matrix was obtained using PROC CORR of SAS software (SAS INSTITUTE, 1997). After the removal of environmental effects, genetic correlations between all wood traits and DBH were estimated (Equation 5) through SELEGEN software (RESENDE, 2002). [Its significances were tested using t-student test (STEEL and TORRIE, 1980, pp 279) (Equation 6).]

$$
\begin{aligned}
& r_{a(x, y)}=\frac{C O \hat{V}_{\hat{\mathrm{a}}(x, y)}}{\hat{\sigma}_{\hat{\mathrm{a}} x} \hat{\sigma}_{\hat{\mathrm{a}} y}} \\
& t=\frac{r_{a(x, y)}}{\sqrt{\frac{1-r_{a(x, y)}^{2}}{n-2}}}
\end{aligned}
$$

Where, $\operatorname{cov}_{\hat{a}(x, y)}$ is the genetic covariance between traits "x" and "y", while $\hat{\sigma}_{\hat{\mathrm{a}} x}$ and $\hat{\sigma}_{\hat{\mathrm{a}} y}$ are the genetic standard deviations (square roots of variances) of trait " $x$ " and trait " $y$ " respectively.

\section{Results}

Variation of wood properties: Average wood property values are presented in Table 2 . Weight loss of heartwood due to fungi ( $T V$ - $H R W$ and $P S-H R W$ ) exhibited the highest coefficient of variation $(C V)$ values, both over

\begin{tabular}{|c|c|c|c|c|c|c|c|c|c|c|}
\hline \multirow{3}{*}{ Wood traits } & \multicolumn{2}{|c|}{$\begin{array}{l}\text { Site effect } \\
\text { (d.f. }=1)\end{array}$} & \multicolumn{2}{|c|}{$\begin{array}{l}\text { Clonal effect } \\
(\text { d. } .=19)\end{array}$} & \multicolumn{2}{|c|}{$\begin{array}{c}\text { (Clone X Site) } \\
\text { (d.f. }=19)\end{array}$} & \multicolumn{2}{|c|}{$\begin{array}{c}\text { Block(Site) } \\
(\text { d.f. }=4)\end{array}$} & \multicolumn{2}{|c|}{ Broad sense heritability } \\
\hline & & $\%$ & & ${ }^{\circ}$ & & 0 & & $\%$ & $\mathrm{II}^{\underline{x}}$ & Standard \\
\hline & $\mathrm{F}$ & VAR & $\mathrm{F}$ & VAR & $\Gamma$ & VAR & $\Gamma$ & VAR & & errot \\
\hline$D B H$ & $3.68^{1: 5}$ & 1.03 & $390^{* *}$ & 30.00 & $1.13^{\mathrm{ns}}$ & 521 & $0.65^{1 \mathrm{~ns}}$ & 0.00 & $0.31 *$ & 0.15 \\
\hline$H W P$ & $3.47^{9 \mathrm{si}}$ & 0.00 & $3.34^{5 . x}$ & 0.00 & $2.02 *$ & 30.92 & $0.70^{=8}$ & 0.00 & $0.02^{\mathrm{ns}}$ & 0.03 \\
\hline$B P$ & $65.46^{* *}$ & 32.51 & $2.28 \div *$ & 12.85 & $0.92 \%$ & 0.00 & $1.33^{\prime \prime s}$ & 0.89 & $0.19^{n s}$ & 0.12 \\
\hline$P P(\log 10)$ & $4.86 *$ & 1.37 & $3.77 * *$ & 30.52 & $1.07 \%$ & 0.00 & $1.57^{* \mathrm{~s}}$ & 2.54 & $0.38 * *$ & 0.17 \\
\hline$I M C$ & $160.4^{* *}$ & 48.06 & $4.90 * *$ & 20.92 & $0.97^{\cdots s}$ & 0.41 & $1.13^{\mu \circ}$ & 0.47 & $0.41^{* *}$ & 0.17 \\
\hline $\operatorname{CH}\left(\mathrm{x}^{2}\right)$ & $142.3^{* *}$ & 43.61 & $2.92 * *$ & 4.99 & $2.07^{*}$ & 13.72 & $2.60^{\mathrm{ws}}$ & 3.41 & $0.09^{\mathrm{ns}}$ & 0.08 \\
\hline$S G(1 / x)$ & $7.53 * *$ & 1.52 & $9.46 \% *$ & 45.26 & $2.27^{* *}$ & 17.36 & $0.80^{-8}$ & 0.00 & $0.47^{* *}$ & 0.19 \\
\hline$V S(\operatorname{Iog} 10)$ & $10.43 * *$ & 4.61 & $7.63 * *$ & 42.72 & $1.84 *$ & 12.17 & $0.83^{\mathrm{ns}}$ & 0.00 & $0.41 * *$ & 0.17 \\
\hline$T S(\log 10)$ & $216.5^{* * *}$ & 44.68 & $7.24 \div$ & 21.40 & $2.28 * *$ & 10.19 & $1.73^{* 8}$ & 1.31 & $0.39 *$ & 0.17 \\
\hline$R S(\operatorname{Iog} 10)$ & $235.2 * *$ & 62.33 & $2.45^{* *}$ & 6.61 & $1.24^{\mathrm{ss}}$ & 2.61 & $0.41^{-8}$ & 0.00 & $0.15^{\mathrm{rs}}$ & 0.10 \\
\hline Ratio T/R $(1$ ogs 10$)$ & $28.21^{* *}$ & 19.76 & $1.08 \mathrm{~ms}$ & 1.99 & $0.84 \%$ & 0.00 & $0.71 \%$ & 0.00 & $0.03^{\mathrm{ns}}$ & 0.05 \\
\hline$L^{*}$ & $10.53^{* *}$ & 3.56 & $10.54 * *$ & 53,38 & 1.53 & 7.71 & $0.75^{-x}$ & 0,00 & $0.56^{* * *}$ & 0.20 \\
\hline$a^{*}(\log 10)$ & $46.75^{* *}$ & 19.44 & $5.1 . * *$ & 31.17 & $1.33 \cdots$ & 6.50 & $0.31=-\ldots$ & 0.00 & $0.36^{* *}$ & 0.16 \\
\hline$b^{*}$ & $76.33 * *$ & 24.22 & $8.66^{* *}$ & 13.05 & $1.04 \%$ & 1.66 & $0.40=$ & 0.00 & 0.57 和视 & 0.20 \\
\hline $\mathrm{E} d(\sqrt{x})$ & $24.25^{* *}$ & 2.95 & $17.08^{* *}$ & 46.26 & $1.44 *$ & 28.83 & $0.83=-x$ & 0.00 & $0.48 * *$ & 0.19 \\
\hline$T V-H R W$ & $323.9 * *$ & 75.87 & $1.21^{\mathrm{ms}}$ & 1.32 & $0.51^{1 ! s}$ & 0.00 & $0.30^{=: 4}$ & 0.00 & $0.05^{\mathrm{n} .}$ & 0.06 \\
\hline$P S-H R W$ & $22.11^{* *}$ & 14.09 & $0.92^{1 \mathrm{si}}$ & 0.00 & $1.42: \%$ & 532 & $1.61=$ & 1.89 & $0.00^{\mathrm{n} \pi}$ & 0.01 \\
\hline$T V-S A P(\log 10)$ & $114.0^{* * *}$ & 50.35 & $1.18^{1 \mathrm{se}}$ & 2.28 & $0.98^{\prime: s}$ & 0.00 & $0.45=$ & 0.00 & $0.01^{\mathrm{n} \varsigma}$ & 0.02 \\
\hline$P S-S A P\left(\mathrm{x}^{15}\right)$ & $73.99 * 4$ & 40.33 & $0.82^{138}$ & 0.00 & $1.12^{\mathrm{s}}$ & 1.92 & $0.42^{\prime *}$ & 0.00 & $0.01^{118}$ & 0.02 \\
\hline
\end{tabular}
$70 \%$. Sapwood weight loss exhibited lower $C V$ values,

Table 3. - Analysis of variance, variance component effects and broad sense heritabilities in 10-year-old Tectona grandis clones from two sites in Costa Rica $(n=110)$.

Legend: ** Statistically significant at $p<0.01$; statistically significant at $p<0.05$, NS: not significantly different.

Abbreviations: $D B H=$ diameter at breast height, $H W P=$ heartwood percentage, $B P=$ bark percentage, $P P=$ pith percentage, $I M C=$ initial moisture content, $G D=$ green density, $S G=$ specific gravity, $V S=$ Volume shrinkage, $T S=$ Tangential shrinkage, $R S=$ Radial shrinkage, Ratio $T / R=$ relation tangential and radial shrinkage, " $L^{*}$ ", " $a^{*}$ " and " $b^{*}$ " are color coordinates, $E d=$ stiffness, $T V-H R W=$ weight loss with Trametes versicolor fungi in heartwood, $P S-H R W=$ weight loss with Pycnoporus sanguineus fungi in heartwood, $T V$-SAP = weight loss with Trametes versicolor fungi in sapwood, $P S-S A P=$ weight loss with Pycnoporus sanguineus fungi in sapwood. 
Table 4. - Phenotypic Pearson (clonal-mean) correlations (below diagonal) and estimated genotypic correlations (above diagonal) in 19 traits $(N=100)$.

\begin{tabular}{|c|c|c|c|c|c|c|c|c|c|c|c|c|c|c|c|c|c|c|c|}
\hline Trait & $D B H I$ & $I T P$ & $B P$ & $P P$ & $I M C$ & (FI) & $S G$ & VS & $T S$ & $R S$ & $\begin{array}{c}\text { Ratio } \\
T / R\end{array}$ & $L^{*}$ & $a^{*}$ & $b^{*}$ & Ed & $\begin{array}{c}T V^{\prime}- \\
H R W\end{array}$ & $\begin{array}{c}P S- \\
H K W\end{array}$ & $\begin{array}{l}T V- \\
S A P \\
\end{array}$ & $\begin{array}{l}P S- \\
S A P \\
\end{array}$ \\
\hline IDI\}III & ] & $0.63^{k \hbar k}$ & $-0.53^{*}$ & $-0.56^{* * k}$ & -0.05 & -0.10 & -0.03 & -0.22 & -0.19 & -0.17 & 0.16 & -0.04 & -0.04 & 0.05 & -0.32 & -0.31 & -0.24 & 0.04 & 0.00 \\
\hline $\mathrm{HWP}^{3}$ & $0.57^{\mathrm{k} \mathrm{k}}$ & 1 & $-0.44^{*}$ & $-0.46^{*}$ & 0.35 & -0.09 & $-0\} .42$ & -0.16 & -10.31 & $-4\} .12$ & -0.20 & 0.08 & -0.12 & 0.14 & $-0.59 * *$ & 0.17 & 0.19 & $0.49 *$ & 0.39 \\
\hline $\mathrm{BP}$ & -0.26 & -0.29 & 1 & 0.20 & 0.20 & $0.52^{-\star}$ & 0.19 & 0.11 & 0.11 & -0.09 & 0.02 & -0.20 & 0.35 & 0.11 & 0.14 & 0.17 & 0.03 & 0.06 & -0.22 \\
\hline$P P$ & -0.45 & -0.18 & -0.05 & 1 & -0.21 & $-10.44 *$ & -0.11 & 0.29 & 0.43 & $0.45^{\star}$ & -0.21 & -0.11 & 0.18 & 0.00 & 0.30 & 0.37 & 0.25 & -0.32 & -0.15 \\
\hline IMC & 0.13 & 0.17 & 0.51 & -0.14 & 1 & 0.32 & $-0.73^{* *}$ & -0.32 & $-0.54^{* * *}$ & $-0.61^{* *}$ & 0.17 & 0.018 & 0.34 & $0.46^{*}$ & $-0.44^{*}$ & 0.24 & -0.19 & 0.34 & 0.39 \\
\hline (i) & 0.16 & 0.10 & 0.60 & $-0.3 \hat{z}$ & $0.75^{*}$ & 1 & 0.41 & -0.15 & -0.17 & -0.43 & 0.14 & -0.21 & 0.40 & $-0,02$ & 0.14 & -0.22 & -0.31 & 0.14 & 0.066 \\
\hline$\$ G$ & -0.02 & 0.15 & -0.02 & 0.20 & -0.49 & -0.23 & 1 & 0.20 & 0.41 & 0.28 & -0.04 & -0.23 & -0.04 & $-0.45^{*}$ & $0.53^{*}$ & -0.40 & -0.39 & -0.23 & -0.31 \\
\hline $\mathrm{VS}$ & -0.15 & -0.18 & 0.24 & 0.11 & 0.10 & 0.15 & -0.08 & 1 & $0.82^{* *}$ & $0.77^{n *}$ & 0.06 & 0.20 & -0.31 & -0.22 & $0.66^{* x+x}$ & 0.38 & 0.18 & -0.07 & -0.17 \\
\hline TS & -0.04 & 0.00 & $0.45^{*}$ & -0.02 & $0.37^{*}$ & 0.44 & -0.05 & 0.50 & 1 & $0.69^{\star}$ & 0.11 & 0.21 & -0.19 & -0.19 & $0.67^{\star *}$ & 0.32 & 0.18 & -0.35 & -0.21 \\
\hline RS & 0.04 & 0.00 & $0.45^{*}$ & -0.03 & 0.39 & 0.46 & -0.01 & 0.43 & $0.69^{*}$ & 1 & -0.33 & -0.07 & -0.23 & -0.40 & $0.52^{* *}$ & 0.29 & 0.00 & -0.09 & -0.19 \\
\hline Ratio $\mathbf{T} \mathbf{R}$ & -0.09 & -0.07 & -0.13 & 0.02 & -0.28 & -0.33 & 0.01 & -0.19 & -0.23 & -0.58 & 1 & 0.30 & -0.07 & 0.35 & 0.16 & -0.06 & -0.15 & -0.28 & 0.12 \\
\hline 1.* & -0.1 .3 & $-(1) .034$ & -0.13 & 0.06 & -0.03 & -10.16 & 0.12 & 0.10 & -0.06 & $-(1) .16$ & 0.15 & 1 & $-0.64^{\mathrm{x}+\mathrm{x}}$ & 0.31 & -0.05 & 0.47 & 0.29 & -0.10 & 0.03 \\
\hline$a^{*}$ & 0.17 & 0.12 & 0.34 & -0.05 & 0.42 & 0.46 & 0.03 & -0.09 & 0.23 & 0.31 & -0.21 & $-0.65^{\star}$ & 1 & 0.15 & 0.08 & -0.24 & -0.30 & -0.19 & -0.09 \\
\hline $\mathrm{b}^{*}$ & 0.16 & 0.35 & 0.23 & 0.01 & 0.51 & 0.33 & -0.31 & -0.03 & 0.27 & 0.29 & -0.18 & 0.014 & $0.35^{* * 3}$ & 1 & -0.28 & $-(1,0) 2$ & 0.37 & -0.03 & -0.16 \\
\hline$E d$ & -0.28 & -0.37 & -0.07 & 0.16 & -0.37 & -0.11 & 0.36 & 0.34 & 0.15 & 0.03 & 0.08 & 0.00 & -0.05 & -0.29 & 1 & -0.07 & -0.25 & $-0.56 * *$ & -0.37 \\
\hline TV-HRW & -0.19 & -0.09 & -0.45 & $0.24-$ & -0.48 & -0.53 & -0.03 & -0.09 & -0.57 & $-0.69 \star$ & 0.34 & $0.3 I^{* * *}$ & $-0.45^{* *}$ & -0.44 & 0.15 & 1 & 0.26 & 0.14 & 0.24 \\
\hline PS-IIRW & -0.01 & 0.12 & 0.031 & 0.06 & 0.27 & 0.12 & 0.18 & 0.03 & 0.29 & 0.29 & -0.14 & 0.013 & 0.05 & 0.29 & -0.25 & -0.29 & 1 & 0.31 & 0.13 \\
\hline 'TV-SAI & -0.26 & -0.04 & -0.35 & 0.11 & -0.45 & -0.48 & -0.0 .3 & -0.10 & -0.53 & -0.50 & 0,20 & 0.17 & -0.38 & $-0,34$ & 0.02 & $0.65^{*}$ & -0.27 & 1 & 0.32 \\
\hline PS-SAP & -0.27 & -0.04 & 0.29 & 0.11 & 0.51 & 0.42 & -0.01 & -0.10 & 0.44 & 0.47 & 0.21 & 0.19 & 0.28 & 0.29 & 0.01 & -0.54 & 0.37 & -0.53 & 1 \\
\hline
\end{tabular}

Legend: numbers in boldface for statistically significant (* denotes $p<0.05$, ** denotes $p<0.01$ ) based on Equation 6 for genotypic correlations. Abbreviations: see Table 3. Phenotypic and genotypic correlation coefficients were estimated with SELEGEN (RESENDE, 2002).

Note: Selegen Software estimates a Bonferroni correction for type 1 error.

from 41 to $51 \%$. $P P$ values registered a large $C V$ of $63 \%$ as well. Meanwhile, color parameters, and $S G$ and $G D$ estimates exhibited the lowest $C V$ values, all below $11 \%$. Some differences were established between Site 1 and Site 2.

Analyses of variance and genetic control: Results of the analyses of variance are presented in Table 3 for all the wood traits and $D B H$. Significant differences $(p<0.01)$ between the two sites were observed for all variables, except $H W P$. The estimated variance component due to site (environment) ranged from 1.4 to $75.9 \%$ of the total ('random-effect') variation (Table 3). The largest site effect (over $50 \%$ ) was exhibited by $T V$-SAP, $R S$ and $T V-H R W$, indicating a strong environmental effect on these traits. In contrast, low site effects (from 14 to $33 \%$ ) were recorded in $P S-H R W$, ratio $T / R, B P$, color coordinates $a^{*}$ and $b^{*}$, while the least site effects were observed for $V S, E d, S G, P P$ and color coordinate $L^{*}$, indicating a stronger genetic control for these traits.

The clonal effect was significant $(p<0.01)$ for $B P, P P$, $I M C, G D, S G$, all shrinkage parameters (except in ratio $T / R$ ), all wood color parameters, and Ed. The color coordinate $L^{*}$ exhibited the highest variance proportion explained by clonal effect, with $53.4 \%$ of its total estimated random variation. For the traits $P P, S V, S G, E d$, and color coordinates $a^{*}$ and $b^{*}$, the estimated clonal variance percentages varied from 20.0 to $46.3 \%$. The results of environmental and clonal effects permit conclude that color in teak could be strongly improved through well sound breeding programs. The lowest across-sites clonal variation was observed in traits $H W P$, ratio $T / R, T V-H R W, P S-H R W, T V-S A P$ and $P S-$ $S A P$.

The interactions between site and clone were significant in $H W P, G D, S G, V S, T S$ and $E d$, contributing with 10 to $31 \%$ to the total estimated variation. Non-significant effects were observed in the remaining wood properties. The block effect was not significant $(p>0.05)$ for all the traits analyzed and contributed $0 \%$ or less than $3.5 \%$ to the total estimated variation. These results may be explained by very homogenous conditions within both. Finally, the error item contributed over $50 \%$ of total variation in traits $B P, P S-S A P, P P, H W P$, ratio $T / R$ and PS-HRW.

Estimated broad-sense heritabilities $\left(\hat{H}^{2}\right)$ ranged from 0.00 to 0.57 (Table 3 ). The highest heritability estimates were recorded for traits $V S$, color parameter $L^{*}, a^{*}$ and $b^{*}, P P, I M C$ and $S G$. These results indicate that these traits are under an important genetic control, with large variation among clones and good prospects for breeding for these traits. Significant estimates of heritability were found for some wood properties, from 0.38 to 0.57 . 
Low values were obtained for $B P, G D, R S$ and ratio $T / R$ $\left(\hat{H}^{2}\right.$ from 0.03 to 0.19 ), and negligible values for $H W P$ and decay resistance of sapwood and heartwood (Table 3).

Phenotypic and genotypic correlations among wood properties: In Table 4 can be seen both, phenotypic (lower diagonal) and estimated genotypic (above diagonal) correlations among all wood properties investigated. Although we used a small study population of clones, there is a evident positive genetic correlation between $D B H$ and $H W P$, as well strong but negative relationship between $D B H$ with both, $B P$ and $P P$. $H W P$ shows a significant negative genetic correlation with $E d$. Interesting to notice is a negative genetic correlation between $S G$ with color factor $b^{*}$ and $E d$. Predictably, there are strong genetic correlations between all three shrinkage properties (TS, $R S$ and VS).

On the other hand, there is a lack of significance $(p>0.05)$ in the correlation between $S G$ and $D B H$, as well as between $I M C$ with $G D, T S$ with $R S, R S$ with $T V$ $H R W, L^{*}$ with $a^{*}$ and $T V-H R W$ with $T V-S A P$. Lack of significance in the genetic correlation $(p>0.05)$ between $S G$ with shrinkage properties (VS, TS or $R S$ ), are also interesting results to be discussed (Table 4). These results suggest that we could improve $S G$ without materially affecting shrinkage. Another strong but predictable genetic correlations involving $S G$ is its strong negative relationship with $I M C\left(\mathrm{r}=-0.73^{* *}\right)$. Therefore, the highest $S G$ values will result low $I M C$. Future research must study this issue precisely, based on a larger genetic sample and a better experimental design.

\section{Discussion}

Sample size utilized in this investigation was the best possible obtained based on the available remaining trees in these trials. Due to the lack of information, the relevance and the possibility of assessing genetic control on teak wood properties, this investigation was conducted. Clonal trials are usually quite homogeneous, however, 6 ramets per clone are at no doubt quite a small sample size.

Average recorded values for $H W P, B P$ and $P P$ are all below expected and reported for fast-grown teak plantations (PÉrez and Kanninen, 2005; Moya and PÉrez, 2008, BHAT, 1995, KoKUTSE et al., 2004). Wood values for $G D, S G$ and shrinkage (VS, TS and $R S$ ), fell between reported values from other studies (MoYA et al., 2003; MoYA and PÉREz, 2008). In relation to wood color parameters, MoYA and BERROCAL (2010) reported similar values in fast-growing teak plantations in Costa Rica. For the case of white-rot fungal attack effect in weight loss, values reported by MoYA and BERROCAL (2010) and BHAT and Florence (2003), BHAT and Indira (2005), Kosutse et al. (2006) and LUKMANDARU and TAKAHASHI (2008) are similar as those reported here with 10 -year-old ramets.

While environment affects almost all wood properties, some of them are under strong genetic control as well, as reported frequently (ZoBEL and JETT, 1995; ZoBEL and van BuiJtenen, 1998). Recently, Moya and PÉRez (2008) found that soil characteristics such as calcium content, cupper, phosphorus, as well as sandy or salty soil texture, correlated with some physical wood properties in teak. Even though there is still little knowledge of site effects on wood properties in tropical clonal forestry, with the exception of eucalyptus (MIRANDA et al., 2007; VANCLAY et al., 2008), there are some reports in minor tree species such as in Dalbergia sissoo (PANDE and SINGH, 2005) in which site conditions significantly affected wood $S G$ and some other wood anatomy properties. However, other studies reported contradictory results, like in Calycophyllum spruceanum (SOTELO et al., 2007), where no site effects were found for any wood property, but some genetic control in wood specific gravity.

The significance of clone $\mathrm{x}$ site interaction, but lack of either main effect for $H W P$, is noteworthy and puzzling. According to ours results, environmental factors affect clones differently in different sites; therefore the site was not affecting all clones similarly. However, this result can be considered with care, because 2 sites only and small ramets quantity were studied.

Some wood properties with important effects in processing and utilization, like drying (related to IMC and all shrinkage properties), mechanical properties (related to SG and Ed), and wood color (based on color coordinates $L^{*}, a^{*}$ and $\left.b^{*}\right)$, exhibited large clonal variation and strong genetic control, for instance, genotypic correlation between $L^{*}$ with $a^{*}$ in this study (Table 4 ). These results support the possibility of including these traits in future teak breeding programs. However, this does not imply abandonment of good silvicultural practices in order to avoid identifiable effects of growth rate on wood properties (ZOBEL and JETT, 1995).

There are some previous studies reporting important genetic control in growth traits in teak (GoH and Monteuuis, 2005; CAllister and Collins, 2008; Solorzano et al., 2012a), but very little on wood properties. Most of the reports are based on $S G$ and $H W P$ (BHAT and INDIRA, 2005; INDIRA and BHAT, 1997; RAO and SHASHIKALA, 2003; SolORZANO et al., 2012a), reporting similar genetic control values to those found in this study. In contrast, RAO and SHASHIKALA (2003) and BHAT and INDIRA (2005) reported lower heritabilities in teak $S G$. RAO and SHASHIKALA (2003) with 16-year-old clonal tests, reported higher heritabilities for $H W P$ than those found in this study.

There is plentiful evidence relating the amount of heartwood in trees to their growing conditions, especially with diameter increments (PÉREZ and KANNINEN, 2003 and 2005; VíQUEZ and PÉREZ, 2005). The best explanations are based on the pipe model (SHINOZAKI et al., 1964, cited by MoRTATAYA et al., 1999), which relates crown growth with diameter increments and the needed sapwood area, and therefore, the resulting heartwood area. In teak, heartwood proportion is a key marketing factor, so it is important to understand its control and possibilities of manipulation. One way of increasing it is through the promotion of higher diameter increments. In this study, we found a clear positive relationship between diameter growth and heartwood proportion (Figure 2a), as reported in similar studies with teak 

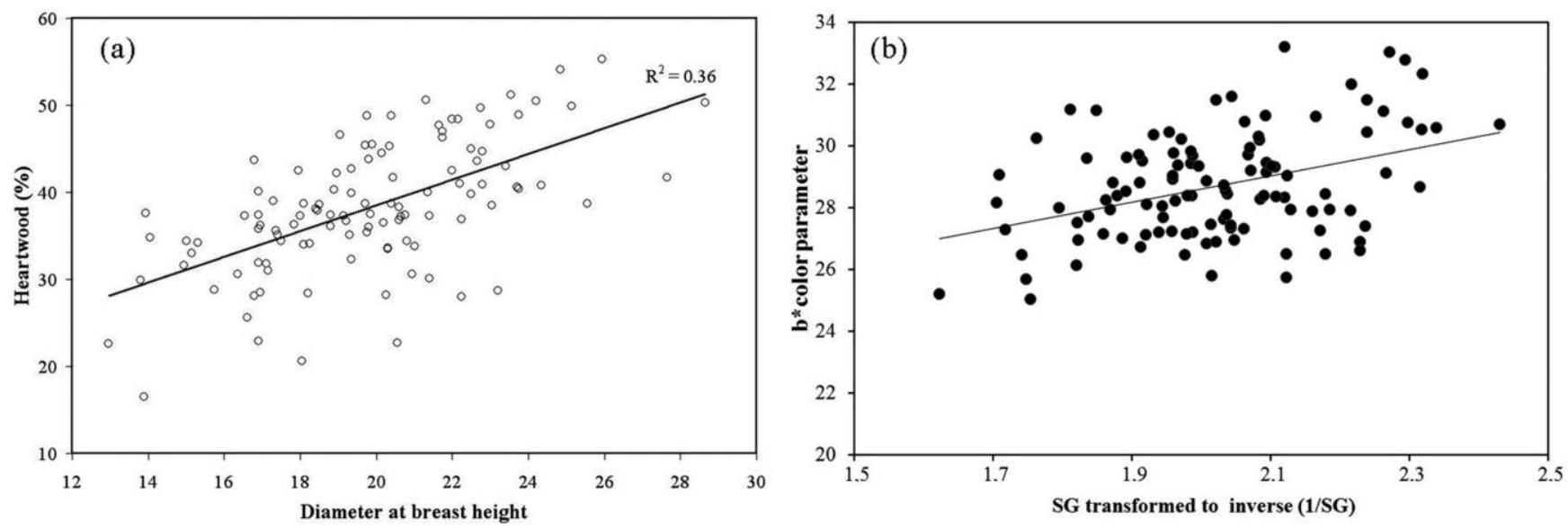

Figure 2. - Phenotypic correlation between heartwood percentage (HWP) and diameter at breast height and between $b^{*}$ color parameter and specify gravity in teak clones tested in northern Pacific from Costa Rica.

(Solorzano et al., 2012a). Since $D B H$ showed a strong clonal variability (Table 3), heartwood proportion could be indirectly improved in teak through selection and use of faster-growing genotypes. Other researchers reported in teak a high to moderate genetic control of $D B H$, with broad-sense heritabilities varying from 0.22 to over 0.50 in early trials (HARSHAP and SOERIANEGARA, 1977; SHARma et al., 2000; CALlister and Collins, 2008). Heartwood formation may be genetically influenced not only by being strongly inherited, but possibly influenced as well by other tree characteristics not included in this investigation. For example, it was demonstrated that heartwood is influenced by site fertility, (MOYA and PÉREZ, 2008), location (KJÆR et al., 1999; PENG et al., 2012), and growing conditions (DERKYI et al., 2010; Moya and Calvo-Alvarado, 2012).

Genetic correlations among traits may have biased effects due to the small sample of trees evaluated. After thinning, slow-growing trees were eliminated from experiment, as well as those with undesirable phenotypic stem characteristics. Reduction of these ramets within clones, tend to produce better vigor estimates for growing traits, but is not necessarily positive in other wood traits, which are clearly losing observations. Coefficient of variation estimates will be downward reduced since they are now based on a smaller number of trees (MATHESON and RAYMOND, 1984) and therefore, it may affect not only genetic correlation estimates, but also in other genetic parameters.

On the other hand, selecting for one of the two wood properties may induce a correlated effect in other related wood properties. Since here we are dealing with clonal estimates, genetic correlations will be involved. However, when for the same two properties there is no evidence of significant genotypic correlations, phenotypic correlations and non-genetic correlations reflecting within-clone statistics must be interpreted as reflecting largely management (environmental) effects (ZoBEL and JETT, 1995). One good example is the relationship between the $D B H$ and $H W P, B P$ and $P P$, in this study. For the clonal teak population $(n=20)$ there is a quite strong positive phenotypic correlation $\left(r=0.57^{* *}\right)$, as reported elsewhere (KJÆR et al., 1999; PÉREZ and KANNINEN, 2003, 2005; VÍQUEZ and PÉREZ, 2005; SOLORZANO et al., 2012b). This is a positive result, since a threshold minimum $H W P$ is required in marketing teak wood. Besides, this relationship was genetically significant (Table 4). This means that if we increase diameter growth through good silvicultural practices, we may increase $H W P$ in teak trees and that if we select trees in a breeding program for higher $D B H$ (Figure 2a), there will be more heartwood and less pith and bark content. Other researchers have established genetic correlations among wood properties in conifers (HANNRUP et al., 2004, CAllister and Collins, 2008) like in our study. Similar studies with tropical tree species, however, are limited (SOTELO et al., 2008).

Very promising for breeding are the positive genotypic and phenotypic correlations between the SG with stiffness ( $r=0.53^{* *}$ and 0.36 respectively). Increasing $S G$ improves mechanical properties. This is a very consistent relationship between these two properties, with a very promising utilization in breeding (ZOBEL and JETT, 1995). On the other hand, $E d$ and $D B H$ did not exhibit significant genetic correlations, but a negative phenotypic correlation $(r=-0.36)$. However, though the phenotypic correlation was significant, it was quite low and may reflect the low number of clones $(n=20)$ and ramets per clone in each site ( $n=2$ to 3 ). Meanwhile, $E d$ and shrinkage also showed a favorable genotypic correlation (Table 4). These correlations can be explained by the shrinkage and $E d$ being correlated with wood density (Ivković et al., 2009). Another important correlation with $E d$ is its negative phenotypic correlation with $H W P$ $(r=-0.59$ ), which indicated that increasing HWP (by tree diameter) would reduce stiffness (lower $E d$ ).

Color is a key factor for marketing teak wood. Therefore, wood color relationships with other wood properties are amongst the most important in this investigation. Interesting to note is the strong negative genotypic correlation estimate of $S G$ with $b^{*}\left(=-0.45^{*}\right)$ (Figure $2 b$ ). This means that increased $S G$ entails decreased yellowness of wood color so darker colour. The $S G$ may be utilized in future as an indirect indicator for recognizing 
wood with better properties. Meanwhile there is a strong genetic correlation between $a^{*}$ and $L^{*}$ parameters $\left(r=-0.64^{*}\right)$. This is a very promising result for breeding since clones with lower $L^{*}$ should produce wood with highest a* values. MOYA and BERROCAL (2010) established through multiple correlation analysis that $D B H$ is the main characteristic that determines $L^{*}$ and $a^{*}$ values in heartwood color in teak trees. SotELo et al. (2008) established significant genetic correlations between $D B H$ and tree height with $b^{*}$ color parameter in Calycophyllum spruceanum. HANNRUP et al. (2004) reported significant genetic correlations between $a^{*}$ and $b^{*}$ color coordinates in Picea abies. However, in this investigation there were no evident association between wood color and DBH.

No genotypic correlation between various wood properties and decay resistance of both sapwood and heartwood was found (Table 4). However, some important properties, such as wood color, can be used as a wooddecay indicator on the basis of phenotype (Table 4). If we look at phenotype correlations for wood-color variables, $b^{*}$ and $a^{*}$ coordinates were negatively correlated with sapwood and heartwood decay under Trametes versicolor. But, these color parameters were positively correlated under Pycnoporus sanguineus wood decay. These color correlations are explained by the fungi's decay effects in the wood, which could be interpreted as an effect from the environmental conditions. MoYA and BERROCAL (2010) found that wood-color variables were differently correlated with decay in different environmental conditions of Costa Rica. They agree with the contention that decay resistance is an effect of environment. It means also that, when decay resistance is not controlled through breeding programs, it could be controlled by environmental conditions.

\section{Conclusions}

These results must be considered as the first ones on teak wood properties genetic control, and therefore treated as non-conclusive. Future investigations are needed in order to validate and contrasts these findings.

This study demonstrates that these teak clones appear to show adequate $S G$, shrinkage and stiffness. The wood color is similar to that of other fast-grown trees. And it also established that variation in $P P, B P$, $I M C, G D, S G$, different shrinkage- and wood-color variables, and Ed is under important genetic control. Estimated broad-sense heritability was from moderate to high for the color parameter $L^{*}$ and VS. Low heritabilities were found in $G D, R S$ and ratio $T / R$ and negligible values were found for $H W P$ and decay resistance in sapwood and heartwood.

Especially interesting results were obtained for $H W P$, since it is a key factor for marketing teak wood. It was estimated that while variation is present between clones, across-sites heritability is very low. There is plentiful evidence relating the amount of heartwood in trees to their growing conditions. However, $H W P$ can be increased with better silvicultural practices, as well as selecting faster-growing trees. It is important to establish the allometric relationships between heartwood and tree-size variables, to give a better understanding of heartwood formation in clonal forestry.

Phenotypic and estimated genetic correlations indicate that in future breeding programs, selecting trees for faster growth will increase heartwood proportion, while reducing pith and bark content. However, fastergrowing trees, through the improvement of $D B H$, should lead to a reduction in wood stiffness and decay resistance. On the other hand, selecting clones with denser wood would improve $E d$ and would reduce yellowness and probably improve wood color. Finally, clone selection with lower $L^{*}$ values could be increase redness $\left(a^{*}\right)$ of wood. However, decay resistance is not controlled strongly by genotype but more by environment.

\section{Acknowledgements}

The authors wish to thank to Vicerrectoría de Investigación y Extensión from Instituto Tecnológico de Costa Rica. Thanks to Precious Woods of Central America (MACORI) for supplying the wood samples for this study, mainly to Eng. Ronald Guerrero and Eng. AdRián Delgado.

\section{References}

ASTM (American Society for Testing and Materials, US) (2003): Standard test methods for specific gravity of wood and wood-base materials. Annual Book of ASTM Standards, Vol. 04.10. Philadelphia. USA (ASTM D 2395-02 and ASTM D 2017-81).

ASTM (American Society for Testing and Materials, US) (2005): Standard Practice for Calculation of Color Tolerances and Color Differences from Instrumentally Measured Color Coordinates. Annual Book of ASTM Standards, Vol. 04.10. Philadelphia. USA (ASTM D 224402 ).

BHAT, K. M. (1995): A note on heartwood proportion and wood density of 8-year-old teak. Indian For 121(6): 514-517.

BhAt, K. M. and E. J. Florence (2003): Natural decay resistance of juvenile teak wood grown in high input plantations. Holzforschung 57: 453-455.

BHAT, K. M. and E. P. INDIRA (2005): Genetic gains in wood quality attributes of clonal teak (Tectona grandis L.f.). J Timber Dev Assoc India 51(1/2): 30-36.

CAllister, A. N. and S. L. Collins (2008): Genetic parameter estimates in a clonally replicated progeny test of teak (Tectona grandis Linn. F). Tree Genet Genome 4: 237-245.

Derkyi, N. S. A., H. Bailleres, G. Chaix, M. F. Thevenon, A. A. Oteng-Amoako and S. Adu-Bredu (2010): Colour variation in teak (Tectona grandis) wood from plantations across the ecological zones of Ghana. Ghana J Forestry 25: 40-49.

GoH, D. K. S. and O. MonteuUis (2005): Rationale for developing intensive teak clonal plantations, with special reference to Sabah. Bois et Forêt des Trop 285: $5-16$.

Hannrup, B., C. Christine, G. Guillaume, M. Grabner, B. Karlsson, I. Le Bayon, J. Lloyd, U. MÜller, H. Pereira, J. C. Rodrigues, S. Rosner, P. Rozenberg, L. Wilhelmsson and R. Wimmer (2004): Genetic parameters of growth and wood quality traits in Picea abies. Scand J Forest Res 19: 14-29. 
HARShAP, R. M. S and I. SOERIANEGARA (1977): Heritability of some characters in teak (Tectona grandis L.f.), pp 649-657. In: Third World Consultation on Forest Tree Breeding, Volume 2. CSIRO, Camberra, Australia.

HunTERLAB (1995): Use measurement of apparence. A welly-Interscience publication, Welly and Sond, New York.

Ivković, M., W. J. Gapare, G. Abarquez, J. Ilic, M. B. Powell and H. Wu (2009): Prediction of wood stiffness, strength, and shrinkage in juvenile wood of radiata pine. Wood Sci Tech 43: 237-257.

INDIRA, E. P. and K. M. BHAT (1997): Variability and heritability of wood density in teak (Tectona grandis L.f.). J Trop For 13(1): 1-5.

KJÆR, E. D., H. R. Siegismund and V. Suangtho (1996): A multivariate study on genetic variation in teak (Tectona grandis (L.). Silvae genet 45: 361-368.

KJÆR, E. D., S. KAJORNSRICHON and E. B. LAURIDSEN (1999): Heartwood, calcium and silica content in five provenances of teak (Tectona grandis). Silvae Genet 48(1): $1-3$.

Kokutse, A. D., A. Stokes, H. Baillères, K. Kokou and C. BAUdAsse (2006): Decay resistance of Togolese teak (Tectona grandis) heartwood and relationship with color. Trees 20: 219-223.

Kokutse, A. D., H. Baillères, S. Stokes and A. KoKou (2004): Proportion and quality of heartwood in Togolese teak (Tectona grandis L.f). Forest Ecol Manage 189: $37-48$.

LUKMANDARU, G. and K. TAKAHASHI (2008): Variation in the natural termite resistance of teak (Tectona grandis L. f.) wood as a function of tree age. Ann For Sci 65: 708-710.

Matheson, A. C. and C. A. Raymond (1984): Effect of thinning in progeny tests on estimates of genetic parameters in Pinus radiate. Silvae Genet 33: 125-128.

Miranda, I., J. Gominho, A. Lourenco and H. Pereira (2007): Heartwood, extractives and pulp yield of three Eucalyptus globulus clones grown in two sites. Appita J 60: $485-488$.

MonteuUis, O. and D. K. S. GoH (1999): About the use of clones in teak. Bois Forêts Trop 261(3): 28-37.

MonteuUis, O. and H. MAÎTRE (2007): Advances in teak cloning: New developments in teak cloning lead to better plantation stock. ITTO Trop For update 17(3): 13-15.

Morataya, R., G. Galloway, F. Berninger and M. KanniNEN (1999): Foliage biomass - sapwood (area and volume) relationships of Tectona grandis L.F. and Gmelina arborea Roxb.: silvicultural implications. For Ecol Manag 113: 231-239.

Mosedale, J. R., B. Charrier and G. JANin (1996): Genetic control of wood colour, density and heartwood ellagitannin concentration in European oak (Quercus petraea and $Q$. robur). Forestry 69: 111-124.

Moya, R. and J. Alvarado-CAlvo (2012): Variation of wood color parameters of Tectona grandis and its relationship with physical environmental factors. Ann Forest Sci 69: 947-959.

MoYA, R. and A. BERROCAL (2010): Wood color variation in sapwood and heartwood of Tectona grandis and its relationship with plantation characteristics, site and decay resistance. Ann For Sci 67: 109.

MoyA, R. and D. PÉrez (2008): Effect of physical and chemical soil properties on physical wood characteristics of Tectona grandis plantations in Costa Rica. J Trop For Sci 20: 147-155.
MoyA, R., D. PÉREZ and V. ARCE (2003): Wood density of Tectona grandis at two plantation spacings in Costa Rica. JTrop For Prod 9(1/2): 153-161.

MuRILlo, O. (2005): Hacia el cultivo de madera en Costa Rica. El Tatascán (Honduras) 17: 17-23.

PANDE, P. K. and M. SiNGH (2005): Inter-clonal, intra-clonal, and single tree variations of wood anatomical properties and specific gravity of clonal ramets of Dalbergia sissoo Roxb. Wood Sci Tech 39: 351-366.

Peng, P., K. LI, K. LiANG and K. XU (2012): A Comparative study on wood properties of teaks from different geographical provenances. Adv Materials Res 430-432: 508-511.

PÉRez, D. and M. Kanninen (2003): Heartwood, sapwood and bark content, and wood dry density of young and mature teak (Tectona grandis) trees grown in Costa Rica. Silva Fennica 37(1): 45-54.

PÉREZ, D. and M. KANNINEN (2005): Stand growth scenarios for Tectona grandis plantations in Costa Rica. Forest Ecol Manage 210: 425-441.

RAO, R. and S. SHASHIKALA (2003): Assessment of growth rate, basic density, heartwood content in select teak clones of CSO. pp 440-447. In: Abstracts Products and Marketing of Teak Wood Products of Planted Forest. Regional workshop products and marketing of teak wood products of planted forest, edited by NAIR, K. V. Bhat and V. Anitha. September 2007. KFRI, Peechi, India.

Resende, M. D.V. (2002): Software Selegen-Reml/Blup. Embrapa Florestas, Colombo, Brasil. 67 pp (Documentos, 77).

RINK, G. (1987): Heartwood color and quality variation in a young black walnut progeny test. Wood Fiber Sci 19(1): 93-99.

SAS InSTITUTE (1997) SAS/STAT ${ }^{\circledR}$ user s guide: version 6.08. Carrey, v.2, 846 p. USA.

Sharma, R., D. Swain and A. K. MANDAl (2000): Estimates of genetic parameters from an open pollinated genetic test of teak (Tectona grandis). J Trop For Sci 2: 44-48.

Solórzano, S., R. Moya and S. Chauhan (2012a): Early genetic evaluation of morphology and some wood properties of Tectona grandis L clones. Silvae genet 61(1-2): 58-65.

SolóRzano, S., R. MoYa and O. MurILlo (2012b): Early prediction of basic density, shrinking, presence of growth stress, and dynamic elastic modulus based on the morphological tree parameters of Tectona grandis. J Wood Sci 58(4): 290-299.

Sotelo, M. C., J. Beaulieu and R. Hernandez (2007): Genetic variation in wood mechanical properties of Calycophyllum spruceanum at an early age in the Peruvian Amazon. Wood Fiber Sci 39: 1-13.

Sotelo, M. C., R. Hernandez, J. Beaulieu and J. C. WEBER (2008): Genetic variation in wood color and its correlations with tree growth and wood density of Calycophyllum spruceanum at an early age in the Peruvian Amazon. New For 35: 57-73.

Steel, R. and J. TORRIE (1980) Principles and Procedures of Statistics. A Biometrical Approach. Second edition. McGraw Hill Book Company. New York, USA.

Thulasidas, P. K., K. M. BHAT T. and OKuma (2006): Heartwood color variation in home garden teak (Tectona grandis) from wet and dry localities of Kerala, India. J Trop For Sci 18: 51-54.

Vanclay, J. K., M. Henson and G. Palmer (2008): Color variation and correlations in Eucalyptus dunnii sawnwood. J Wood Sci 54: 431-435. 
Varghese, M., A. Nicodemus, P. K. Ramteke, G. AnbaZHAGi, S. S. Bennet and K. Subramanian (2000): Variation in growth and wood traits among nine populations of teak in Peninsular India. Silvae Genet 49: 201-205.

VíqueZ, E. and D. PÉREZ (2005): Effect of pruning on tree growth, yield, and wood properties of Tectona grandis plantations in Costa Rica. Silva Fennica 39: 381-390.
XAVieR, A. and W. C. ОтоNi (2009): Applications of micropropagation of Eucalyptus in clonal silviculture in Brazil. Agronomía Costarricense 33: 303-307.

ZoBeL, B. J. and J. B. JETT (1995): Genetics of wood production. Springer Verlag, Berlin, Germany.

ZoBeL, B. J. and J. P. vAN BUIJTENEN (1998): Wood Variation: its causes and control. Springer Verlag, Berlin, Germany.

Herausgeber: Johann Heinrich von Thünen-Institut. Bundesforschungsinstitut für Ländliche Räume, Wald und Fischerei. Schriftleitung: Institut für Forstgenetik, Sieker Landstrasse 2, D-22927 Grosshansdorf

Verlag: J. D. Sauerländer's Verlag, Berliner Strasse 46, D-63619 Bad Orb Anzeigenverwaltung: J. D. Sauerländer's Verlag, Bad Orb

Gesamtherstellung: PPPP Norbert Wege e.K., Gladenbach — Printed in Germany. 